\title{
Artigos
}

\section{O estudo de prevalência de deficiências e incapacidades como instrumento de planejamento das atividades de atenção à saúde e reabilitação no Programa Saúde da Família*}

\author{
The study of prevalence of handicap and \\ impairment as an instrument of planning \\ activities concerning health and rehabilitation \\ in Family Health Program
}

\author{
Eucenir Fredini Rocha ${ }^{1}$, Ana Rita de Paula ${ }^{2}$, Márcia Regina Kretzer ${ }^{3}$
}

\begin{abstract}
ROCHA, E.F.; PAULA, A. R. de; KRETZER, M. R. O estudo de prevalência de deficiências e incapacidades como instrumento de planejamento das atividades de atenção à saúde e reabilitação no Programa Saúde da Família. Rev. Ter. Ocup. Univ. São Paulo, v.15, n.1, p. 1-10, jan./abr., 2004.

RESUMO: São parcos os estudos acerca de prevalência de deficiências e incapacidades na América Latina e no Brasil. Entre os poucos existentes temos os estudos multicêntricos de prevalência patrocinados pelo Ministério da Saúde em 11 municípios brasileiros, com metodologia proposta pela Organização Panamericana de Saúde (OPS). Esses estudos, fundamentados na Classificação Internacional de Deficiências, Incapacidades e Desvantagens (CIDID), apresentam dificuldades na sua aplicação e no planejamento de ações em territórios adscritos, pois são realizados por amostragem. $\mathrm{O}$ atual artigo apresenta dados da experiência do estudo de prevalência do PSF/Fundação Zerbini, na região Sudeste do município de São Paulo, com coleta censitária nas famílias cadastradas. A coleta foi realizada por agentes comunitários de saúde das equipes de família (médico, enfermeiro, auxiliar de enfermagem) e equipe de reabilitação (fisioterapeuta, fonoaudiólogo e terapeuta ocupacional) do território estudado, buscando-se dessa forma, uma relação íntima dos pesquisadores com a realidade retratada. A própria enquete está sendo utilizada como um instrumento dos profissionais no planejamento das ações preventivas e na assistência à população em saúde e reabilitação. Como objetivo geral, o estudo pretende estabelecer um diagnóstico complexo, relacionando dados de prevalência, da caracterização do território e dados referentes à exclusão social dessa população visando o planejamento de ações de intervenção.
\end{abstract}

DESCRITORES: Estudos transversais. Pessoas deficientes/reabilitação. Planejamento em saúde. Reabilitação. Saúde em família.

\footnotetext{
* Apresentação modificada do pôster apresentado no VII Congresso Brasileiro de Terapia Ocupacional, Porto Alegre, RS, outubro de 2001 .

${ }^{1}$ Profa. Dra. do Departamento de Fisioterapia, Fonoaudiologia e Terapia Ocupacional da FMUSP. Mestre em Psicologia EscolarIPUSP e Doutora em Psicologia Social-IPUSP.

${ }^{2}$ Psicóloga, Mestre em Psicologia Social e Doutora em Psicologia Clínica pela Universidade de São Paulo, Consultora do Ministério da Saúde para a área de atenção à pessoa com deficiência e da Sorri Brasil.

${ }^{3}$ Enfermeira, Coordenadora Regional do PSF/QUALIS - Fundação Zerbini, Região Sudeste do município de São Paulo.

Endereço para correspondência: Departamento de Fonoaudiologia, Fisioterapia e Terapia Ocupacional da Faculdade de Medicina da Universidade de São Paulo. Rua Cipotânea, 51. Cidade Universitária. CEP 05360-000. São Paulo, SP. e-mail: eucenir@usp.br, arpa123@terra.com.br, mrkretzer@terra.com.br
} 


\section{INTRODUÇÃO}

$\mathrm{A}$ partir da aprovação, pela ONU, do Programa de Ação Mundial para as Pessoas com Deficiência (1982) a atenção à pessoa com deficiência no sistema público brasileiro vem sendo inserida aos poucos nos programas realizados na área da saúde. Dessa forma, o documento "Atenção à pessoa portadora de deficiência no Sistema Único de Saúde: planejamento e organização de serviços", do Ministério da Saúde (1993) ${ }^{(1)}$ passou a garantir prevenção, reabilitação e igualdade de oportunidades à pessoa portadora de deficiência.

Ainda nas décadas de 80 e 90 , temos as primeiras experiências afinadas, de uma forma geral, com as proposições internacionais para inserir a reabilitação e a atenção à saúde da pessoa com deficiência nos sistemas locais de saúde no Brasil. Alguns exemplos disso, no Estado de São Paulo, são os serviços implantados pela Secretaria do Estado da Saúde de São Paulo, em 1983; pela Secretaria Municipal da Saúde de São Paulo, em 1991/1994 (OLIVER, 1998) e pela Secretaria Municipal de Saúde de São José dos Campos, em 1996/1999 (ROCHA; DE PAULA, 1999). Em 2000, temos a implantação, na cidade de São Paulo, da primeira proposta de serviços de reabilitação no Programa da Saúde da Família, através da Secretaria de Saúde do Estado de São Paulo e de seus parceiros: Casa de Saúde Santa Marcelina e Fundação Zerbini (ROCHA et al., 2001).

Uma das maiores dificuldades encontradas pelos gestores de diferentes experiências referentes à Atenção à Saúde da Pessoa com Deficiência, no Brasil, tem sido o planejamento de ações compatíveis com as reais necessidades da população com deficiências e/ou incapacidades, pois existem poucos estudos epidemiológicos aplicados às realidades locais, o que limita a definição dos recursos humanos e dos materiais necessários, bem como o planejamento de estratégias de intervenção.

\section{Estudos de prevalência das deficiências e in- capacidades no Brasil}

Em 1992, a Associação Fluminense de Reabilitação, patrocinada pelo Ministério da Saúde, realizou o primeiro Estudo de Prevalência de Incapacidades no município de Niterói-RJ (MAIOR et al., 1994), posteriormente a mesma pesquisa foi realizada em mais 10 municípios brasileiros $^{(2)}$.

Esses estudos seguiram a metodologia proposta pela OPS, desenvolvida a partir da comparação de diferentes estudos de prevalência e incapacidades realizados entre 1961 e 1975, em 18 países da América Latina e Caribe, com resultados que variaram de $1 \%$ a $15 \%$ (MAIOR et al., 1994). As diferenças nos resultados foram atribuídas, em grande parte, às diferentes metodologias utilizadas e aos diversos conceitos de incapacidade, o que evidenciou a necessidade da criação de um protocolo único que pudesse ser aplicado em diferentes locais.

Essa necessidade foi atendida com a estruturação da Classificação Internacional de Deficiências, Incapacidades e Limitações - ICIDH (OMS, 1989) ${ }^{(3)}$, organizada pela OPS em 1990 (MAIOR et al., 1994; SÃO JOSÉ DOS CAMPOS, 1999). Esse protocolo foi aplicado em alguns países da América Latina e também no Brasil. Nos municípios citados, o estudo foi coordenado pela Associação Fluminense de Reabilitação e gestores municipais, com o apoio financeiro do Ministério da Saúde ${ }^{(4)}$.

Conforme a Associação Fluminense (MAIOR et al., 1994), a proposta da OPS é uma pesquisa por amostragem, em municípios de médio porte (cerca de 500.000 habitantes), com perguntas agrupadas em itens e subitens da CIDID e que deve ser realizado em duas fases. A primeira fase consiste de uma enquete domiciliar, aplicada em casas sorteadas. As perguntas não são diretas e são feitas por meio de repetição, fugindo tanto quanto possível de perguntas estigmatizantes como

\footnotetext{
(1) A proposta do documento é de subsidiar o planejamento e a organização de serviços dentro do Sistema Único de Saúde, definindo ações e funções de reabilitação a serem desenvolvidas nos diferentes níveis de atendimento à população. Os públicos-alvo do documento são os gerentes e coordenadores locais de saúde, objetivando subsidiar a capacitação de pessoal e instrumentalizar a implantação nos programas locais de saúde.

(2) Cidades de médio porte (por volta de 500.000 habitantes)/porcentagem em relação ao total da população residente. Brasília -DF (2,81\%); Campo Grande - MS (4,8\%); Canoas - RS (4,30\%); Feira de Santana - BA (9,60\%); Maceió - AL (6.97\%); Niterói - RJ (7.50\%); Santo André - SP (7,05\%); Santos - SP (6,11\%); São José dos Campos - SP (5,90\%); Silva Jardim - RJ $(6,18 \%)$; Taguatinga - DF $(4,00 \%)$.

(3) Essa classificação que foi revista nos últimos cinco anos e resultou na CIF - Classificação Internacional de Funcionalidade, Incapacidade e Saúde (CIF, 2003).

(4) A metodologia utilizada na pesquisa da Associação Fluminense difere totalmente das utilizadas pelo IBGE nas suas diferentes aplicações (1981, 1991 e 2000).
} 
"tem paralisia”, “é cego", "é surdo" etc... Assim, os pesquisadores perguntam se alguém na residência tem "dificuldades de se locomover" "falta parte do corpo", "dificuldade de enxergar mesmo de óculos", “dificuldade para distinguir palavras e sons” etc. Há também questões mais inespecíficas que enquadram situações da velhice ou mesmo pequenas deficiências parciais que não provocam incapacidades (MAIOR et al., 1994, p. 37-8).

Os dados colhidos no questionário devem ser validados, posteriormente, na fase dois do estudo, por uma equipe de técnicos de reabilitação treinados para a avaliação técnica de uma amostragem das pessoas com "suspeita" de ter alguma deficiência ou incapacidade, proporcionando assim, elementos consistentes para o resultado final.

Outro aspecto a ser ressaltado nessa proposta de estudo refere-se ao fato de o questionário destacar a importância da CIDID, centrando os resultados nas incapacidades e não nas deficiências ${ }^{(5)}$.

Os resultados desses estudos, nos diferentes municípios em que foram aplicados, forneceram dados capazes de sinalizar a direção a ser seguida pelos serviços de saúde ao indicar a predominância de um ou vários tipos de deficiências e/ou incapacidades, tais como limitações músculo-esqueléticas, problemas psicológicos ou de linguagem, entre outros. Esses dados, porém, são genéricos e não localizam as pessoas com deficiências e incapacidades nos diferentes micro-territórios de cada município.

A própria Associação Fluminense de Reabilitação destacou alguns problemas em relação ao protocolo aplicado: 1) o questionário é pobre em relação às causas sociais da problemática; 2) sua aplicação necessita de um treinamento e acompanhamento intenso da equipe aplicadora e uma forte adesão da comunidade, o que nem sempre é possível; 3) a proposta é muito complexa e cara para ser incorporada em todo o país, e propõe, assim, que sejam realizadas adaptações locais do modelo.

Com a rediscussão da CIDID e sua reorganiza- ção na CIF - Classificação Internacional de Funcionalidade, Incapacidade e Saúde (2003), o modelo proposto de estudo epidemiológico está sujeito a revisões.

A experiência de estudo de prevalência censitário de deficiências e incapacidades, do Programa de Saúde da Família - Fundação Zerbini na região de Vila Prudente e Sapopemba do município de São Paulo

A atenção à saúde da pessoa com deficiência no Programa da Saúde da Família do município de São Paulo.

Em abril de 2000, a Fundação Zerbini - na época parceira da Secretaria de Saúde do Estado (SES) ${ }^{(6)}$ de São Paulo e responsável pela implantação e gerenciamento do Programa Saúde da Família (PSF) Fundação Zerbini e Norte do município de São Paulo solicitou a participação do REATA - Laboratório de Estudos em Reabilitação e Tecnologia Assistiva da FMUSP na elaboração de um projeto de atenção à saúde e reabilitação da pessoa com deficiência para ser implementado nas unidades básicas de saúde sob sua responsabilidade. A proposta foi organizada e expandida para a região leste da cidade, no PSF, sob a responsabilidade da Casa de Saúde Santa Marcelina, também parceira da SES-São Paulo. A implantação ocorreu em outubro do mesmo ano, com a contratação das duas equipes de reabilitação(7).

O Programa Saúde da Família traz a discussão da atenção integral à família no atendimento realizado pelas equipes de saúde compostas por um médico, um enfermeiro, um ou dois auxiliares de enfermagem e cinco ou seis agentes comunitários ${ }^{(8)}$. Os atendimentos são realizados através de visitas domiciliares e consultas feitas nas unidades de saúde da família (USF). Especificamente no PSF - Fundação Zerbini e Casa de Saúde Santa Marcelina, há também o acompanhamento e as consultas das equipes de saúde da pessoa com deficiência, saúde mental e saúde bucal, ambulatórios de especialidades e Casas de Parto.

\footnotetext{
(5) O fato de centrar em incapacidades, já aponta para um novo modelo de intervenção em reabilitação que não está centrado na patologia/doença e deficiência e sim numa observação do usuário a partir do conceito de alteração do "desempenho" e seu estado de saúde, conceitos que viriam ser reforçados com a CIF (2003).

(6) Desde o inicio de 2003 a parceria se dá com a Secretaria Municipal de Saúde de São Paulo, quando ocorreu o processo de municipalização dos serviços de saúde.

(7) Para a Fundação Zerbini: três fisioterapeutas, três fonoaudiólogos e três terapeutas ocupacionais alocados na região sudeste (Vila Prudente e Sapopemba) e na região Leste do município de São Paulo, gerenciado pela Casa de Saúde Santa Marcelina, nove fisioterapeutas, cinco fonoaudiólogos e cinco terapeutas ocupacionais.

${ }^{(8)}$ Cada equipe de saúde da família atende em média 1000 famílias, sendo que cada agente comunitário de saúde é responsável por cerca de 250 famílias. As visitas a essas famílias são mensais, acompanhadas de protocolos específicos, delimitados pelo Ministério da Saúde, de controle de condições de saúde dessas pessoas.
} 
A partir de uma abordagem interdisciplinar com um enfoque comunitário, compreende-se saúde para além dos aspectos puramente biológicos, considerando-se também as implicações sociais, culturais, políticas e econômicas. As ações desenvolvidas objetivam diminuir os riscos à saúde, prevenir doenças, acompanhar, intervir e encaminhar o mais rápido possível os pacientes e melhorar as condições de saúde da população em geral. Dentro desta perspectiva, a atenção à saúde da pessoa com deficiência no Programa Saúde da
Família - SP objetiva a inclusão dessa população em todos os níveis assistenciais e a sua inclusão em outros serviços e atividades da comunidade.

Para tanto, o estudo de prevalência de deficiências e incapacidades se tornou prioritário para a elaboração dos atendimentos, nos diferentes territórios cobertos pelas equipes do PSF e de reabilitação (Tabela 1), visando o aprofundamento da discussão das possibilidades de intervenção no campo social e o desmonte dos processos de exclusão.

Tabela 1 - População abrangida pelo projeto dentro do PSF - Fundação Zerbini e Casa de Saúde Santa Marcelina.

\begin{tabular}{lcccc}
\hline Regiões da Cidade de São Paulo & $\begin{array}{l}\text { Unidades de } \\
\text { saúde }\end{array}$ & Equipes de família & Total de famílias & $\begin{array}{c}\text { População total } \\
\text { (aproximada) }\end{array}$ \\
\hline Leste - Casa de Saúde Santa Marcelina & 26 & 89 & 89.000 & 356.000 \\
Sudeste- Fundação Zerbini & 9 & 33 & 39.600 & 146.662 \\
Sudeste - Secretaria de Saúde do Estado & 01 & 06 & 6.000 & 24.000 \\
TOTAL & 41 & 128 & 134.600 & 538.400 \\
\hline
\end{tabular}

Cabe ressaltar que a pesquisa completa, em sua primeira fase, só foi desenvolvida no PSF - Vila Prudente e Sapopemba - Fundação Zerbini e são alguns dos resultados do período de janeiro de 2001 a outubro de 2002 que iremos apresentar adiante.

Os objetivos do estudo de prevalência no Programa da Saúde da Família - Fundação Zerbini

Todos os objetivos do estudo epidemiológico foram discutidos com as equipes de saúde da família e de reabilitação e pretendeu-se com os dados: 1) concluir o diagnóstico situacional da atenção em saúde e em reabilitação da população em pauta no território definido; 2) planejar detalhadamente as intervenções; 3) determinar as necessidades de recursos humanos, materiais e tecnológicos para as diferentes ações nas diferentes áreas do território abrangido. Outra finalidade do estudo foi avaliar a qualidade e a eficiência da assistência prestada, ou seja, o grau de melhora clínica, autonomia e participação social desse segmento populacional. O conjunto dos dados poderá ainda, subsidiar outras propostas de implantação de serviços na área de Programas de Saúde da Família.

Para a realização da pesquisa, o grupo responsável (REATA, Coordenação do PSF / Fundação Zerbini) adaptou a metodologia da OPS para um estudo censitário simplificado, possível de ser aplicado rapidamente pelos Agentes Comunitários de Saúde (ACSs). Elaborou-se, com vistas ao acompanhamento qualitativo e quantitativo da assistência, um Subsistema de Informação, regional, em complementaridade ao SIAB - SUS (Sistema de Informação na Atenção Básica do Sistema Único de Saúde). Para tanto, utilizou-se a CIDID - Classificação Internacional de Deficiências, Incapacidades e Desvantagens (1989). Foram elaborados os seguintes instrumentos de informação:

- fichas para identificação de suspeitos de deficiência a serem aplicadas pelos Agentes Comunitários (F1);

- roteiros a serem aplicados pela equipe de reabilitação para avaliação e validação de deficiência e incapacidades intelectual, psicológica, de audição e linguagem, neurológica infantil e adulto. Não foram elaborados os seguintes roteiros: deficiência visual, músculo-esquelética, sensitivas, gerais e generalizadas;

- ficha de acompanhamento de caso baseada na classificação de tipo e graus de Incapacidades e Desvantagem, a ser utilizada pela equipe de Reabilitação, Para avaliar e caracterizar a situação inicial da clientela. Se aplicado sistematicamente, com intervalo de tempo de no mínimo três meses, esse instrumento possibilita analisar o grau de melhora alcançado em termos de incapacidades e desvantagem e, conseqüentemente, a qualidade da assistência prestada (F2);

- ficha de acompanhamento de caso pelo Agente Comunitário de Saúde, com periodicidade quadrimestral, a fim de avaliar alterações na participação familiar e social e na redução das condições incapacitantes e de desvantagem, além do 
acompanhamento das condições gerais de saúde (F3);

- ficha de produtividade das equipes de reabilitação, por tipo de atendimento realizado, para analisar e avaliar os princípios e modalidades de assistência quantitativa e qualitativa $(\mathrm{F} 4)$.

O Subsistema de Informação foi implantado parcialmente: as fichas F1 e F4 foram utilizadas integralmente e continuam em uso; as fichas F2 e F3 não foram aplicadas por dificuldades operacionais e administrativas no PSF. Já os roteiros de avaliação foram utilizados parcialmente e muitos casos foram avaliados a partir da experiência clínica da equipe de reabilitação. Atualmente estuda-se a utilização da CIF (2003), em caráter experimental.

O objetivo da implantação do subsistema de informação foi permitir estudos comparativos com o SIAB, tais como: incidência e prevalência de casos com deficiências decorrentes de hipertensão arterial (Acidentes Vasculares Cerebrais) ou de Diabetes (cegueira, amputação, neuropatias entre outros), que ainda não ocorreram e deverá ser desenvolvido na segunda fase desse estudo que está em elaboração.

Como proposta de fluxo de informações, o subsistema de informação teve como finalidade: 1) levantar os suspeitos de deficiências através dos Agentes Comunitários de Saúde; 2) encaminhar essas pessoas para as equipes de saúde da família para discutir o caso e avaliar a necessidade de intervenções específicas da equipe de saúde e de reabilitação da pessoa com deficiência; 3 ) a equipe de reabilitação avaliar através de protocolo sistematizado os casos encaminhados pelas equipes de saúde da família- ESFs; 4) realizar o seguimento específico de reabilitação; 5) realizar o acompanhamento sistemático, na comunidade e na família, pelos ACSs, através da ficha F3 para detectar a qualidade da melhora clínica e a inserção social da pessoa.

\section{A detecção de pessoas com deficiências ou incapacidades na comunidade: a metodologia e o processo}

Este trabalho é o resultado da aplicação das fichas para identificação de suspeitos de deficiência pelos Agentes Comunitários (F1) e do acompanhamento dos usuários no período de janeiro de 2001 a outubro de 2002 pelas ESFs e da equipe de reabilitação através da ficha F4.
A ficha F1 foi resultado de um processo de discussão e experimentação de diferentes possibilidades de coleta de informações junto à comunidade.

Primeiramente, o questionário proposto pela OPS e utilizado nas pesquisas patrocinadas pelo Ministério da Saúde, foi simplificado e apresentado para as equipes de saúde da família, que consideraram impossível sua aplicação devido ao tamanho e tempo que tomariam, tornando difícil, assim, a enquete junto às famílias.

Após os testes, optou-se pela elaboração de um protocolo bem simplificado, que pudesse servir, simultaneamente, para a detecção de pessoas com deficiência e como instrumento educativo para os profissionais das equipes de saúde da família (ESF). É importante destacar que esses profissionais tinham muitas dúvidas e um grande desconhecimento sobre a abrangência, as características e as possibilidades de intervenções de saúde na população deficiente. Com o treinamento para a aplicação do protocolo $(\mathrm{F} 1)$, várias questões foram discutidas, ou seja, a pesquisa tornou-se o momento introdutório das ESFs nas especificidades da problemática da deficiência e serviu para que a equipe de reabilitação se envolvesse com as questões gerais da saúde pública, muitas vezes, distante de sua formação.

Assim, após a elaboração de um protocolo simplificado, que passou a ser chamado de a Ficha dos Bonecos (F1) - pois uma parte de seus dados foi coletada por meio da visualização de uma figura humana e, a outra parte, através de um questionário bem simplificado e com um espaço livre para observações não previstas —, detectou-se várias pessoas com suspeita de deficiências e incapacidades que foram encaminhadas para as equipes de atenção à saúde da pessoa com deficiência para avaliação e validação da hipótese.

É importante ressaltar que muitos casos foram encaminhados para a equipe de reabilitação de saúde da família antes do protocolo ser implementado. Este fato ocorreu concomitantemente ao processo de treinamento dos ACSs para a aplicação da ficha F1. Cabe observar ainda, que os instrumentos de levantamento de suspeitos foram divididos por faixas etárias: de 0 a 14 anos e 15 anos ou mais, respeitando as características de cada fase ${ }^{(9)}$.

Depois de realizado um pré-teste com a aplicação de 50 protocolos, foram feitas as alterações necessárias no instrumento. Em seguida foram impressas 15.000 fichas, referente a cerca de $10 \%$ da população local (146.662.000 habitantes), para serem

(9) A divisão das fichas em duas faixas etárias (0-14 anos e 15-50 anos ou mais) seguiu o padrão dos registros do SIAB. 
aplicadas em todos os domicílios.

As fichas F1 foram compostas da seguinte forma:

- dados gerais: nome da unidade de saúde, número da família, endereço, nome da pessoa, sexo, idade, data do preenchimento, área, microárea, nome do Agente Comunitário de Saúde;

- dados registrados através da figura do boneco: 1) ausência (falta alguma parte do corpo: perna, braços, língua, orelha, órgãos etc.); 2) dor (dor intensa ou moderada, a longo tempo ou recentemente em alguma região do corpo, ouvido, olhos, pernas etc.); 3) alterações de movimentos (alteração, ausência, dificuldade, diminuição ou excesso do movimento de alguma região do corpo); 4) alterações de sensibilidade (não sente ou sente em demasia alguma região do corpo ou rosto, formigamento, pele muito quente ou muito fria, alteração da cor); 5) deformidades (algum tipo de alteração de alguma parte do corpo, inclusive boca, nariz); 6) uso de aparelhos e acessório (ex: bolsa de ostomia, cadeira de rodas, muleta, andador, aparelho auditivo, goteira, tala, outros). Esses dados foram registrados na figura e especificados conforme $o$ entendimento do ACSs e informante;

- perguntas específicas para crianças ou adolescentes (0-14 anos): tem o corpo muito mole, tem corpo muito duro; permanece sempre na mesma posição; tem dificuldade em pegar e soltar objetos; tem mais de 4 meses e não sustenta a cabeça; tem mais de 4 meses e não acompanha objetos com os olhos; tem mais de 6 meses e não senta sozinha; tem mais de 9 meses e não engatinha; tem mais de 1 ano e 5 meses e não anda sozinha; engasga constantemente ao se alimentar; tem dificuldade de ouvir; tem mais de 3 anos e não brinca em grupos; sente enjôos e desmaios; muito agitada/agressiva consigo e com os outros; tem dificuldade para falar, não fala, troca letras, gagueja; não reage, assusta, chora, faz caretas, quando ouve barulhos fortes; tem algum problema de visão (vesgo, não vê); tem alguma dificuldade para aprender, para ler ou escrever; baba constantemente; tem problemas de coordenação das mãos; tem dificuldades de equilibrar-se ou locomover-se de forma normal; engasgos ao engolir; tem problema respiratório grave; tem problema de voz (muito fraca, muito grossa, perde a voz;

- perguntas específicas para jovem, adulto e idoso (15-
50 anos ou mais): tem dificuldade para vestir-se, limpar-se, comer e ir ao banheiro sozinho; tem algum problema respiratório grave; tem problemas de comportamentos (estranhos, absurdos etc.); engasga constantemente ao se alimentar; tem dificuldade para reconhecer e/ou nomear objetos, paisagens, pessoas; não reage ou reconhece sons, tem dificuldade para ouvir; tem alguma dificuldade para aprender, ler ou escrever; baba constantemente; tem perda de consciência ou convulsões; tem problemas de memória; tem problema de voz (muito fraca, muito grossa, perde a voz); tem desmaios ou enjôos freqüentes; tem algum problema de visão (vesgo, não vê, secreções, avermelhamento); tem dificuldade para falar, não fala, troca letras, gagueja; tem problemas de coordenação das mão; tem dificuldades de equilibrar-se ou locomover-se de forma normal.

Os ACSs foram treinados pela equipe de reabilitação para a aplicação do protocolo nos domicílios. A partir da hipótese de deficiência os casos foram encaminhados para as ESFs, que discutiu as prioridades de avaliação e de intervenção ${ }^{(10)}$ de reabilitação. Para a validação dos casos com deficiência, a equipe de reabilitação baseou-se na CIDID.

Esses procedimentos resultaram num banco de dados que foram tabulados em diferentes categorias, como veremos adiante, e servem atualmente para o encaminhamento da pesquisa e para a organização de procedimentos básicos de reabilitação adequados à realidade de cada Unidade de Saúde da Família.

Como continuidade da proposta de estudo epidemiológico está em elaboração a segunda fase da pesquisa, agora, fundamentada na CIF (2003).

\section{RESULTADOS}

As autoras julgam importante ressaltar que não consideram a pesquisa como um fato isolado do processo de implantação do serviço. Assim sendo, os dados colhidos devem ser sempre compreendidos à luz do momento da implantação da atenção à saúde da pessoa com deficiência em cada uma das equipe de saúde da família, ao mesmo tempo em que a sua análise está influenciando a compreensão e a tomada de decisões no transcorrer do trabalho. A pesquisa é então compreendida como não acabada e como instrumento de qualificação e aprimoramento dos profissionais envolvidos.

\footnotetext{
(10) Paralelo a aplicação da pesquisa o serviço de reabilitação foi sendo implantando, com atendimentos em domicilio, Unidade de Saúde da Família e ambulatório.
} 
Com a aplicação da Ficha F1 (Ficha dos Bonecos) pelos ACSs e os outros encaminhamentos sem as fichas, foram detectadas 4476 pessoas com um ou mais tipos de deficiências distribuídas, conforme o gráfico 1, nas 9 Unidades Básicas de Saúde do PSF/Zerbini, no período de janeiro de 2001 a outubro de 2002.

As deficiências e incapacidades foram agru- padas a partir das categorias da CIDID, a saber: dor, deficiências neurológicas infantil e adulta, deficiências da linguagem, deficiência auditiva, deficiência visual, deficiências gerais, deficiência intelectual, deficiências múltiplas, deficiências estéticas, deficiências músculoesqueléticas, deficiências generalizadas, deficiências metabólicas e outras deficiências (Gráfico 1).
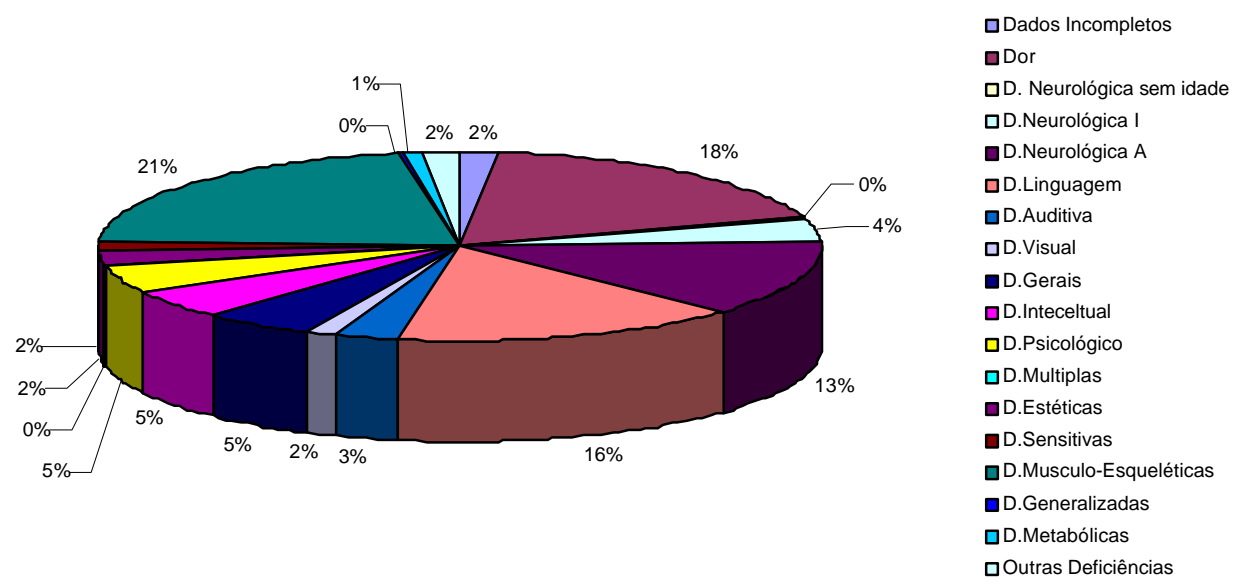

Gráfico 1 - Distribuição da amostra segundo prevalência ( $n=4476$ pessoas no total da área de abrangência).

As deficiências metabólicas fazem parte das deficiências gerais, porém foram destacadas para estudos posteriores (cegueira, amputações etc). As deficiências neurológicas também foram separadas em infantil e adulto visando outros estudos, como, por exemplo, para detectar casos de AVC decorrentes de hipertensão arterial e os casos de paralisia cerebral decorrentes de situações de risco na gravidez.

Do total de pessoas detectadas com deficiências ou incapacidades, 39\% são homens e $60,8 \%$ são mulheres. A predominância da faixa etária ficou entre 15 e 59 anos, em $56 \%$ da população detectada. Entre 0 a 14 anos, foram detectados $15,3 \%$ de pessoas e, com mais de 60 anos, 25,2\%. Cerca de 3,4\% das fichas estavam com dados incompletos.

A partir desses dados podemos identificar dois fenômenos relevantes, ou seja, a prevalência de deficiências no sexo feminino e na faixa etária adulta, no seu período produtivo. A predominância de mulheres pode ser explicada, entre diversas razões, por ser esta a população que se encontrava em casa no momento da enquete. Já, em relação a indicação da faixa etária (no período produtivo), provavelmente, isto se deve ao processo de desgaste gerado pela má qualidade de vida e pelas condições de trabalho dessa população.
Os índices apresentados no Gráfico 2 são os resultados da divisão da prevalência pela população adstrita a cada unidade básica de saúde.

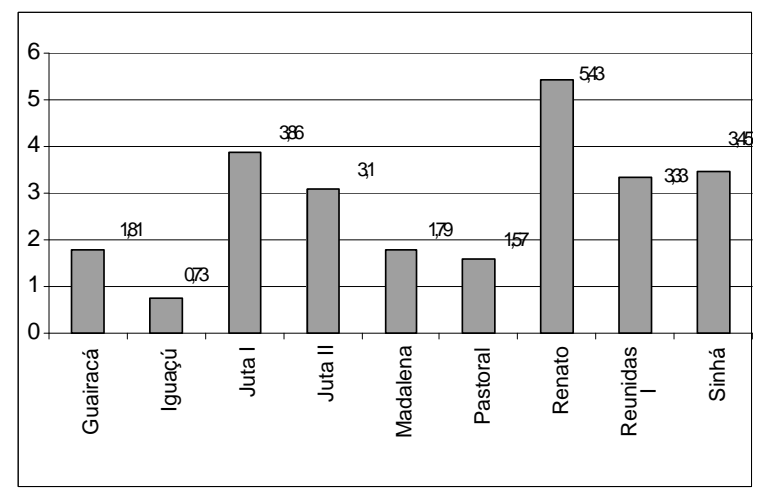

Gráfico 2 - Prevalência de suspeitos por unidade. Índice médio por unidade ( $\mathrm{n}$ = área de abragência de cada unidade).

As diferenças nos índices médio de prevalência por unidades refletem as diferenças no processo de envolvimento com a pesquisa das equipes de saúde da família e do conjunto de ACSs, bem como a diversidade demográfica (faixa etária prevalente da população), as características socioeconômico presentes em cada área e microáreas de abrangência. Algumas 
Unidades Básicas de Saúde têm em seu território a predominância de uma população mais idosa, como, por exemplo, a do Guairacá e a do Reunidas. Já em outras Unidades Básicas de Saúde podemos ter a predominância de uma população mais jovem, pois esses dados têm relação com a história do bairro e origem da população. Além das diferenças por faixa etária, temos características diferentes determinadas pela renda per capita diferenciada, condições de moradia, de saneamento e de acesso a bens materiais e culturais. Essas diferenças influenciam o perfil epidemiológico de cada área.

Apresentamos aqui um dos exemplos de prevalência que surgiu como significativa na coleta dos dados por tipo deficiência: a dor (Gráfico 3).

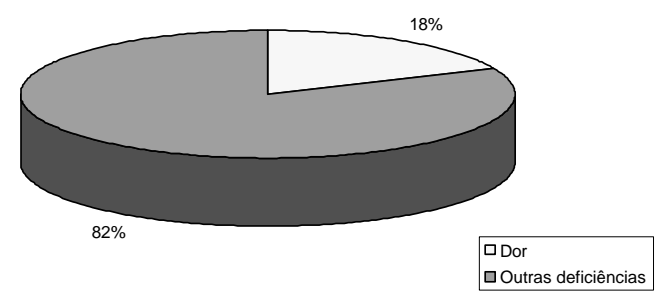

Gráfico 3 - Prevalência da dor em relação a outros tipos de deficiências.

Ela aparece como o maior índice de incapacidade referida, quer transitória, quer crônica, $18 \%$. Sua predominância também ocorre entre as mulheres, ou seja, em $66 \%$ delas e entre $34 \%$ dos homens. De início esse dado está servindo para o estabelecimento de um diálogo mais intenso com a equipe de saúde mental, uma vez que muitos casos têm associado quadros clínicos nessa área, como, por exemplo, a depressão. Outro dado interessante é a sua incidência entre as mulheres na faixa etária do climatério. Esse campo merece um maior aprofundamento, com análises comparativas a outros estudos epidemiológicos da dor.

A partir do encaminhamento de alguns casos de dor às equipes de saúde da família, essas pessoas estão sendo inseridas em atividades grupais de caminhada e outros trabalhos corporais, tais como alongamento, relaxamento, danças circulares, entre outros, coordenadas por profissionais da reabilitação ou estagiários de terapia ocupacional e com a participação de ACSs, dependendo da finalidade e composição do grupo.

Assim, como a dor, cada tipo de deficiência detectado necessita ser relacionado a condições gerais epidemiológicas de cada região. Esse estudo detalhado está sendo desenvolvido junto às equipes de saúde de família e coordenado pela equipe de reabilitação. Como próximo passo, os dados coletados deverão ser transpostos para a CIF (2003) e cruzados com outros indicadores do SIAB.

É importante ressaltar que essa modalidade de coleta de dados proporciona nome e endereço de cada pessoa com deficiência ou incapacidade, permite conhecer a sua história de vida, saber os outros procedimentos de saúde que o PSF, ou eventualmente outro serviço de saúde, está lhe prestando, bem como subsidia as equipes de saúde de reabilitação em termos do que é emergente, prioritário e necessário desenvolver em relação a ações preventivas e reabilitacionais nas famílias e comunidades locais.

Em novembro de 2002, através da tabulação dos dados registrados na ficha de produtividade (F4), foi possível avaliar os avanços da implantação da atenção à saúde da pessoa com deficiência no PSF e as contribuições do estudo de prevalência (Quadro).

\begin{tabular}{|c|c|c|}
\hline Ação & $\begin{array}{l}\mathbf{N}^{0} \text { Absoluto } \\
\text { de pessoas }\end{array}$ & $\%$ \\
\hline 1. Casos discutidos com as Equipes de Saúde da Família & 4476 & $100 \%$ \\
\hline \multicolumn{3}{|l|}{ 2. Casos avaliados pela equipe de saúde da pessoa } \\
\hline com deficiência e reabilitação & 2315 & $52 \%$ \\
\hline 3. Casos que não necessitavam de intervenção reabilitacional (do total de avaliados - 2315) & 193 & $8,3 \%$ \\
\hline 4. Casos encaminhados para intervenção reabilitacional (do total de avaliados -2315 ) & 1893 & $82 \%$ \\
\hline \multicolumn{3}{|l|}{ 5. Casos que se encontravam em lista de espera para a } \\
\hline primeira intervenção (do total de avaliados - 1893) & 441 & $23 \%$ \\
\hline 6. Casos que se encontravam em lista de espera para outras intervenções (do total de avaliados -1893 )* & 111 & $5,8 \%$ \\
\hline 7. Casos desligados / faltosos para avaliação (do total de avaliados -2315$)^{* *}$ & 111 & $4,8 \%$ \\
\hline 8. Casos desligados / faltosos em atividades de intervenção reabilitacional (do total de avaliados -1893 )** & 303 & $16 \%$ \\
\hline 9. Casos que tiveram alta do serviço PSF (do total de avaliados - 1893) & 176 & $9,2 \%$ \\
\hline $\begin{array}{l}\text { 10. Casos que tiveram alta da equipe de profissionais de atenção à saúde e reabilitação da pessoa com } \\
\text { deficiência (do total de avaliados - 1893) }\end{array}$ & 58 & $3 \%$ \\
\hline 11.Óbitos (do total de avaliados - 1893) & 139 & $7,3 \%$ \\
\hline 12.Migração para outro serviço de reabilitação (do total de avaliados - 1893) & 172 & $9 \%$ \\
\hline
\end{tabular}

* exames clínicos, consultas com especialistas, entre outras.

** pessoas que foram consideradas autônomas para se deslocarem até a Unidade Básica de Saúde para o atendimento e que faltaram.

Casos graves, com dificuldades de locomoção passaram a ser atendidos em domicílio. 
É importante ressaltar que nem todas pessoas com deficiência ou alguma incapacidade necessitam de reabilitação e cada caso deve ser discutido com as equipes de saúde da família, pois outras ações de saúde podem e devem ser desenvolvidas para essa população.

Entende-se que a análise dos dados necessita de aprofundamento e, como continuidade ao trabalho de pesquisa, o grupo coordenador dessa primeira coleta de dados (REATA - Laboratório de Estudos em Reabilitação e Tecnologia Assitiva do curso de Terapia Ocupacional da USP e Coordenação Técnica do PSF - Fundação Zerbini) está estabelecendo uma parceria com o Centro Colaborador da OMS para Família de Classificações Internacionais/Faculdade de Saúde Pública da USP para o desenvolver a segunda fase da pesquisa.

Como objetivos gerais desse segundo momento do estudo tem-se:

1. conhecer as diferentes concepções de deficiência existentes no território abrangido pelo projeto, a saber: da população/familiares e equipes de saúde da família;

2. delinear o perfil do território abrangido pelas nove Unidades Básicas de Saúde do PSF/Fundação Zerbini, em termos de características geográficas, demográficas, culturais, sócioeconômicas e de saúde para identificar as características do território que potencializam ou não o desempenho das atividades do cotidiano pessoal e social das pessoas com deficiência e incapacidade na área abrangida pelo PSF/ Fundação Zerbini;

3. realizar o diagnóstico das condições de saúde no território de abrangência do PSF/Fundação Zerbini, através da análise do Banco de Dados, do período de 2001 a 2003, e realizar um estudo epidemiológico da incidência de doenças potencialmente incapacitantes e a tendência no período de 2000/ 2004, tais como hipertensão e diabetes;

4. realizar um estudo piloto para validar a aplicação da Classificação de Funcionalidade, Incapacidade e Saúde- CIF como instrumento de avaliação da deficiência. O mesmo instrumento deverá ser aplicado em 18 pacientes atendidos na área de abrangência do Programa de Saúde da Família - Fundação Zerbini da Secretaria Municipal de Saúde de São Paulo pelo período de um ano.
A participação das equipes continuará a ser intensa visando, principalmente, o retorno dos dados para o planejamento de ações de saúde, preventivas e reabilitacionais na população com deficiências e incapacidades.

\section{CONCLUSÕES}

A realização de estudos de prevalência em territórios específicos de Unidades Básicas de Saúde é um instrumento valioso na elaboração de ações de saúde, prevenção e reabilitação para a população com deficiências e incapacidades. Estudos censitários são eficientes para a elaboração de estratégias específicas de mudança de perfil epidemiológico.

Outra consideração importante, decorrente da experiência do PSF/QUALIS - Fundação Zerbini da Secretaria Municipal de Saúde de São Paulo é que os estudos de prevalência devem ser desenvolvidos em estreita relação com a prática assistencial e não se constituir meramente em pesquisas isoladas e pontuais.

Como possibilidades de ação decorrentes desses estudos, as equipes de saúde e de reabilitação podem:

- cruzar os dados colhidos com dados epidemiológicos do PSF e do SIAB;

- elaborar planejamento específico nas UBSs. Os resultados demonstram que cada unidade de saúde possui uma especificidade epidemiológica, social, cultural e econômica. Os dados de prevalência determinam o planejamento das ações locais e específicas para a realidade de cada território e não a implementação programas verticalizados e predeterminados, que muitas vezes não tem impacto local;

- associar as ações de reabilitação a outras ações de saúde desenvolvidas nas Unidades Básicas de Saúde, quer sejam preventivas, educativas ou curativas.

E, finalmente, o estudo ora apresentado demonstra a necessidade de equipes de reabilitação na rede básica para mudar o perfil epidemiológico das deficiências e incapacidades. 
ROCHA, E.F.; PAULA, A. R. de; KRETZER, M. R. The study of prevalence of handicap and impairment as an instrument of planning activities concerning health and rehabilitation in Family Health Program. Rev. Ter. Ocup. Univ. São Paulo, v.15, n.1, p. 1-10, jan./abr., 2004.

\begin{abstract}
S: The investiments in studies on the handicapped and impaired citizens in Latin America and Brazil are rather scarce. On these few occasions, there are multicentric studies about prevalence sponsored by Ministry of Health in 11 Brazilian cities, with methodology proposed by Panamerican Organization of Health (OPS). These studies, supported by the International Classification of Handicap, Impairment and Disadvantage (CIDID), present difficulties in their applications and in their action planning in nearby territories, since they are carried out by samples. The current article presents data based on experiences about the studies of prevalence of PSF/Zerbini Foundation in the southwest region of Sao Paulo, with the collect from credentialized families. The collect was made by health agents of family team community (such as doctor, nurse, nursing assistant) and team of rehabilitation (physiotherapist, speech therapist and occupational therapist ) of the studied territory, trying to set up in this way a relationship between the researcher and the related reality. The investigation itself has been used as an instrument by the professionals in the planning of preventive actions as well as in the health and rehabilitation assistance to population. As the general aim, the study intends to establish a complex diagnoses relating data about prevalence, about the characterization of territory and data about social exclusion of this population in order to plan action of intervention.
\end{abstract}

KEYWORDS: Cross-sectional studies. Disabled persons/rehabilitation. Health planning. Rehabilitation.

\title{
REFERÊNCIAS
}

BRASIL. Ministério da Saúde. Coordenação de Atenção a Grupos Especiais. Programa de Atenção à Saúde da Pessoa PoutadodedDeficîncia.Atenção à pessoa portadora de deficiência no Sistema Único de Saúde: planejamento e organização dos serviços. Brasília: Secretaria de Assistência à Saúde, 1993.

CIF - CLASSIFICAÇÃO INTERNACIONAL DE FUNCIONALIDADE, INCAPACIDADE E SAÚDE. Centro Colaborador da Organização Mundial de Saúde para a Família de Classificações Internacionais, Org.; coordenação da tradução Cássia Maria Buchalla. São Paulo: EDUSP, 2003.

MAIOR, I. L.; TEIXEIRA, M. A.; EMYGÏDIO, R. F.; HOELZ, T. S. (Coord.). Estudo de prevalência de incapacidades no município de Niterói. Niterói: Associação Fluminense de Reabilitação, 1994.

OLIVER, F.C. Saúde mental e saúde da pessoa com deficiência: estudo do processo de incorporação de assistência pelos serviços municipais de saúde numa região do Município de São Paulo (1989-1995). São Paulo, 1998. Tese (Doutorado) - Faculdade de Saúde Pública, Universidade de São Paulo.

OMS - ORGANIZAÇÃO MUNDIAL DA SAÚDE. Classificação internacional das deficiências, incapacidades e desvantagens (handicaps): um manual de classificação das conseqüências das doenças. Lisboa, 1989.
ONU - ORGANIZAÇÃO DAS NAÇÕES UNIDAS [1982]. Programa de ação mundial para as pessoas com deficiência. São Paulo: CEDIPOD - Centro de Documentação e Informação do Portador de Deficiência, 1992.

ORGANIZACIÓN PANAMERICANA DE LA SALUD. Determinación de prevalencia de discapacidades. Manual de encuestas domiciliarias. Ginebra: Oficina Sanitária Panamericana, Oficina Regional de la Organizacion Mundial de La Salud,1990.

ROCHA, E. F.; DE PAULA, A. R. Avaliação da implantação da política de reabilitação no município de São José dos Campos - período de janeiro/97 a dezembro/99. São Paulo, 1999. [mimeografado].

ROCHA, E. F.; DE PAULA, A. R.; KRETZER, M. R.; DA SILVA, L. B. Avaliação da implantação do "Programa de atenção à saúde da pessoa portadora de deficiência $\mathrm{e}$ reabilitação no PSF/QUALIS nas regiões leste e sudeste do Município de São Paulo. Outubro de 2000 a dezembro de 2001”. São Paulo, 2001.

SÃO JOSÉ DOS CAMPOS. Secretaria Municipal da Saúde. Coordenação de Especialidades de Saúde. Estudo de prevalência de incapacidades no município de São José dos Campos. SP. São José dos Campos: Associação Fluminense de Reabilitação, 1999. [mimeografado]. 